\title{
Editorial
}

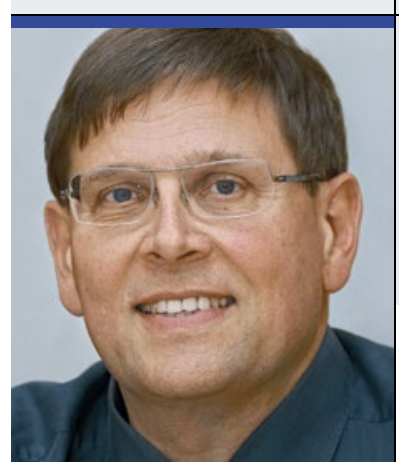

"Die Versorgung chronischer Schmerzpatienten muss

überwiegend ambulant und wohnortnah verfügbar sein. "

\section{Patientenversorgung im Mittelpunkt}

$\mathrm{D}$ as Schwerpunktthema des Deutschen Schmerzund Palliativtages 2016 lenkt den Blick auf das Zentrum aller ärztlichen Bemühungen. Menschen mit Erkrankungen - oft in schwierigen Lebenslagen - zu versorgen ist die zentrale Aufgabe in Praxis, Ambulanz und Krankenhaus.

Unser Gesundheitssystem - eines der besten der Welt - hat für die Versorgung ein abgestuftes System geschaffen, das von der ambulanten wohnortnahen Betreuung bis zur hochspezialisierten stationären Einrichtung allen Patienten die adäquate und notwendige Versorgung bieten soll. Über die Bedarfsplanung wird die ambulante und stationäre Versorgung sichergestellt. Ausgerechnet für eines der häufigsten Krankheitsbilder, die chronischen Schmerzen (nach neuesten Daten 23.000.000 Betroffene in Deutschland, davon 2.800 .000 mit schwersten problematischen Schmerzerkrankungen), existieren derartige gesicherte Versorgungsformen nicht.

\section{Versorgungsdefizite}

Wie sehr die Versorgung von Patienten mit chronischen Schmerzen mehr von Zufälligkeiten als von verlässlichen Strukturen geprägt ist, wurde auf dem 1. Nationalen Versorgungsforum Schmerz deutlich, zu dem die Deutsche Schmerzliga (DSL) gemeinsam mit der Deutschen Gesellschaft für Schmerzmedizin (DGS) und dem Berufsverband der Ärzte und Psychologischen Psychotherapeuten in der Schmerzund Palliativmedizin in Deutschland (BVSD) am 12.11.2015 nach Berlin eingeladen hatte.

Alle Beteiligten - Gesundheitspolitik, Kassenvorstände, Kassenärztliche Bundesvereinigung und Ärztekammer - betonten die Notwendigkeit einer Neuordnung der schmerzmedizinischen Versorgung, in der eine sorgfältige Bedarfsplanung die Grundlage für eine Sicherstellung der Versorgung darstellt. Dabei herrschte Übereinstimmung, dass diese Versorgung überwiegend ambulant und wohnortnah verfügbar sein muss - dass also Sie, die diesen Patienten jeden Tag in Ihren Praxen und Ambulanzen begegnen, hierbei die entscheidende Rolle spielen.

\section{Abgestufte Schmerzversorgung}

Die schmerzmedizinische Versorgung erfordert ein abgestuftes Vorgehen. Entscheidende Bedeutung kommt der hausärztlichen und der fachärztlichen Versorgungsebene zu. Hier werden die Weichen gestellt um Schmerzchronifizierung zu verhindern, hier müssen und können Risikofaktoren identifiziert und adäquate Therapien eingeleitet werden. Das hierfür nötige aktuellste Wissen über Grundlagen, Diagnostik und Therapie vermittelt der Deutsche Schmerz- und Palliativtag 2016 mit dem Schwerpunkt "Schmerzmedizin in der Hausarztpraxis" in zahlreichen Workshops und Symposien.

\section{Netzwerke entscheiden}

Für die schwierigsten Patienten mit weit fortgeschrittener Chronifizierung sind ohne Frage Schmerzmediziner mit einer umfassenden (nicht nur fachgebietsgebundenen) Qualifikation erforderlich, die ein multimodales Therapieangebot erlauben. In Ermangelung gesicherter, abgestufter schmerzmedizinischer Versorgungsstrukturen ist hier nicht nur Ihr Wissen, sondern sind auch Ihre Qualitäten als Netzwerker gefragt.

Lassen Sie uns gemeinsam daran arbeiten, dass das Jahr 2016 für unsere Patienten mit chronischen Schmerzen und auch für Sie selbst, die diese Patienten versorgen, ein gutes Jahr wird. Hierfür wünsche ich Ihnen viel Glück, Kraft und Zuversicht!

Herzlichst, Ihr

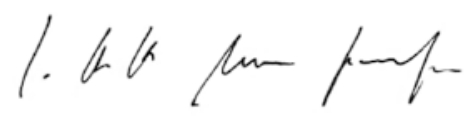

Gerhard H.H. Müller-Schwefe 